\title{
Differentially Expressed Gene Profile of Acanthamoeba castellanii Induced by an Endosymbiont Legionella pneumophila
}

\author{
Eun-Kyung Moon ${ }^{1} \odot$, So-Min Park², Ki-Back Chu², Fu-Shi Quann ${ }^{1,3}$, Hyun-Hee Kong ${ }^{4, *}$ (i) \\ 'Department of Medical Zoology, Kyung Hee University School of Medicine, Seoul 02447, Korea, ${ }^{2}$ Department of Biomedical Science, Graduate \\ School, Kyung Hee University, Seoul 02447, Korea, ${ }^{3}$ Medical Research Center for Bioreaction to Reactive Oxygen Species and Biomedical Science \\ Institute, School of Medicine, Graduate School, Kyung Hee University, Seoul 02447, Korea, ${ }^{4}$ Department of Parasitology, Dong-A University College \\ of Medicine, Busan 49201, Korea
}

\begin{abstract}
Legionella pneumophila is an opportunistic pathogen that survives and proliferates within protists such as Acanthamoeba spp. in environment. However, intracellular pathogenic endosymbiosis and its implications within Acanthamoeba spp. remain poorly understood. In this study, RNA sequencing analysis was used to investigate transcriptional changes in $A$. castellanii in response to $L$. pneumophila infection. Based on RNA sequencing data, we identified 1,211 upregulated genes and 1,131 downregulated genes in A. castellanii infected with L. pneumophila for $12 \mathrm{hr}$. After $24 \mathrm{hr}$, 1,321 upregulated genes and 1,379 downregulated genes were identified. Gene ontology (GO) analysis revealed that $L$. pneumophila endosymbiosis enhanced hydrolase activity, catalytic activity, and DNA binding while reducing oxidoreductase activity in the molecular function (MF) domain. In particular, multiple genes associated with the GO term 'integral component of membrane' were downregulated during endosymbiosis. The endosymbiont also induced differential expression of various methyltransferases and acetyltransferases in $A$. castellanii. Findings herein are may significantly contribute to understanding endosymbiosis of $L$. pneumophila within $A$. castellanii.
\end{abstract}

Key words: Acanthamoeba, Legionella, endosymbiosis, differential gene expression

Acanthamoeba spp. is one of the most abundant protozoan in the environment and commonly isolated from soil and water. Acanthamoeba spp. trophozoite usually feeds on bacteria, fungi, algae or small organic particles by phagocytosis [1]. However, some bacteria have developed strategies to resist phagocytosis, survive intracellularly and exploit Acanthamoeba spp. for multiplication [2]. These bacteria are able to survive in encysted Acanthamoeba spp. which protects the endosymbionts from adverse environmental conditions [3]. Acanthamoeba spp. not only enables the endosymbionts to persist in the environment but also enhances its pathogenicity [4]. Moreover, since mammalian macrophages and amoebae show similar interactions with endosymbionts, investigating the endosymbiotic relationship between intracellular pathogens and Acanthamoeba spp. would contribute to understanding how these organisms

\footnotetext{
- Received 14 October 2020, revised 15 December 2020, accepted 21 December 2020. *Corresponding author (hhkong@dau.ac.kr) (c) 2021, Korean Society for Parasitology and Tropical Medicine This is an Open Access article distributed under the terms of the Creative Commons Attribution Non-Commercial License (https://creativecommons.org/licenses/by-nc/4.0) which permits unrestricted non-commercial use, distribution, and reproduction in any medium, provided the original work is properly cited.
}

behave in the mammalian cells and its evasion of the human immune system.

Acanthamoeba spp. can be a host for a wide range of pathogenic microorganisms such as Legionella pneumophila, Chlamydophila pneumoniae, Cryptococcus neoformans, Mycobacterium avium, Listeria monocytogenes, and Pseudomonas aeruginosa, etc [3,5-7]. Among these microorganisms, the interaction between Acanthamoeba spp. and Legionella spp. is one of the most investigated. After uptake of Legionella spp. by Acanthamoeba spp., Legionella spp. forms a specialized compartment called Legionella-containing vacuole (LCV). LCV avoids fusion with lysosomes to deter lysosomal digestion and also inhibits phagosomal maturation, thereby enabling $L$. pneumophila to actively replicate inside the LCV [8].

To date, LCV and a large number of effectors transferred by the intracellular multiplication/defective organelle transport (Icm/Dot) type IV system of Legionella spp. have been identified [9-12]. Although the roles of these genes from Legionella spp. have been evaluated, little research has been done on genes of Acanthamoeba spp. during endosymbiosis with Legionella spp. To understand the intracellular survival strategy of 
Legionella spp., inhibition of phagosome lysis in Acanthamoeba spp. needs to be studied. In this study, total transcriptional changes of A. castellanii in response to survival and replication of $L$. pneumophila during $12 \mathrm{hr}$ and $24 \mathrm{hr}$ were investigated by RNA sequencing analysis.

The LCV in the Legionella-infected A. polyphaga has been reported to remain intact for up to $8 \mathrm{hr}$ post-infection (hr pi), disrupted by $12 \mathrm{hr}$ pi, and eventually lysed to release the intracellular pathogens into the cytoplasm of the amoeba by 18 to $24 \mathrm{hr}$ pi [13]. L. pneumophila infection incurred the lysis of more than $80 \%$ of A. polyphaga at $24 \mathrm{hr}$ pi, and it has also been suggested that the intracellular condition may significantly differ between $12-18 \mathrm{hr}$ pi and $24 \mathrm{hr}$ pi [13]. Contrary to the previous findings, L. pneumophila-infected A. castellanii in the present study remained intact even at $24 \mathrm{hr}$ pi. Therefore, gene expression patterns at $12 \mathrm{hr}$ pi and $24 \mathrm{hr}$ pi were compared to confirm whether drastic differences were present in L. pneumophila-infected A. castellanii at these 2 time points.

A. castellanii was infected with L. pneumophila [14], and the Legionella-infected Acanthamoeba (L+A) was incubated for 12 $\mathrm{hr}$ and $24 \mathrm{hr}$ at $25^{\circ} \mathrm{C}$ incubator. mRNA-Seq reads were mapped using TopHat software [15], and differentially expressed gene were determined based on BEDtools and EdgeR [16-18]. And we used the FPKM (fragments per kilobase of exon per million fragments) as the method of determining the expression level of the gene regions. Gene classification was based on searches done by DAVID (http://david.abcc.ncifcrf.gov/).

RNA samples from different experimental conditions were sequenced to investigate the endosymbiosis-induced gene expression changes in A. castellanii (Fig. 1). A total of 7,108 genes whose expressions changed $12 \mathrm{hr}$ pi and $24 \mathrm{hr}$ pi were displayed using a heat map (Fig. 1A). Genes from each group were colorized based on their expression level. Strongly upregulated/ downregulated genes, as indicated by intense red/blue colors, were more prevalent in the $12 \mathrm{hr}$ pi group than the $24 \mathrm{hr}$ pi group. Genes whose expression levels changed more than 2 fold were selected for further analysis (Fig. 1B). Among the 7,018 genes, 1,211 and 1,131 genes in the $12 \mathrm{hr}$ pi group were upregulated and downregulated more than 2 fold, respectively. Similarly, 1,321 and 1,379 genes from the $24 \mathrm{hr}$ pi group were upregulated and downregulated more than 2 fold, each respectively. Venn diagram revealed that a fraction of the DEGs found in the $12 \mathrm{hr}$ pi overlapped with the DEGs from $24 \mathrm{hr}$ pi group (Fig. 1C). Our results revealed that 2,342 and 2,700 DEGs in $L$. pneumophila-infected A. castellanii at $12 \mathrm{hr}$ pi and $24 \mathrm{hr}$ pi were changed more than 2 fold, respectively. More DEGs were observed at $24 \mathrm{hr}$ pi than at $12 \mathrm{hr}$ pi, which may indicate that more gene involvement is required for survival of $L$. pneumophila in the later stages of infection.

DEGs of L. pneumophila-infected A. castellanii were assigned an Entrez Gene ID and subsequently classified into 3 domains: biological process (BP), cellular component (CC), and molecular function (MF). Classified DEGs were subdivided further into various gene ontology (GO) terms under each of the domains. In the $12 \mathrm{hr}$ pi group, DEGs were assigned to 10 subcategories in BP, 8 subcategories in CC, and 23 subcategories in MF (Fig. 2). During the $12 \mathrm{hr}$ endosymbiosis, 17 genes in 'regulation of transcription' (domain: $\mathrm{BP}$ ), 30 genes in the 'nucleus' (domain: CC), and 37 genes in 'DNA binding' (domain: MF) were determined to be the most upregulated genes. Within the CC domain, 200 downregulated genes were involved in the $\mathrm{GO}$ term 'integral component of the membrane'. In the $24 \mathrm{hr}$ pi group, DEGs were subdivided into 29, 13, and 41 GO terms under BP, CC, and MF domains, each respectively (Fig. 3). In the BP domain, 26, 39, and 32 genes from the GO terms 'regulation of transcription', 'intracellular signal transduction', and 'cyclic nucleotide biosynthetic process' were drastically downregulated, each respectively. In the CC domain, similar to the 12 hr pi A. castellanii, 270 downregulated genes were involved in the GO term 'integral component of membrane'. In the MF domains, downregulated genes associated with the GO terms 'zinc ion binding' and 'protein kinase activity' were 57 and 42 , respectively. From each of the domains, 26 genes from 'carbohydrate metabolic process' (domain: BP), 30 genes from the 'nucleus' (domain: CC), and 40 genes from 'DNA binding' (domain: $\mathrm{MF}$ ) were mainly upregulated.

DEGs from A. castellanii, which were upregulated or downregulated more than 10 fold post-infection with $L$. pneumophila, were listed in Tables 1 and 2. In the $12 \mathrm{hr}$ pi A. castellanii, 47 out of 1,211 DEGs were upregulated (Table 1) and 90 out of 1,131 DEGs were downregulated more than 10 fold (Table 2). Identities for several most upregulated proteins in this group were 2 hypothetical proteins (1,100 fold and 345 fold), S-adenosylmethionine-dependent methyltransferases (173 fold), and GDPD-mannose-3', 5'-epimerase (87 fold) (Table 1). GO analysis of the assigned Entrez Gene IDs revealed that the DEG which underwent 1,100 fold increase was a hypothetical protein that belonged to the DNA binding (GO: 0003677) category. Similarly, the GDPD-mannose-3',5'-epimerase which was increased 87 fold, was associated with catalyt- 
A

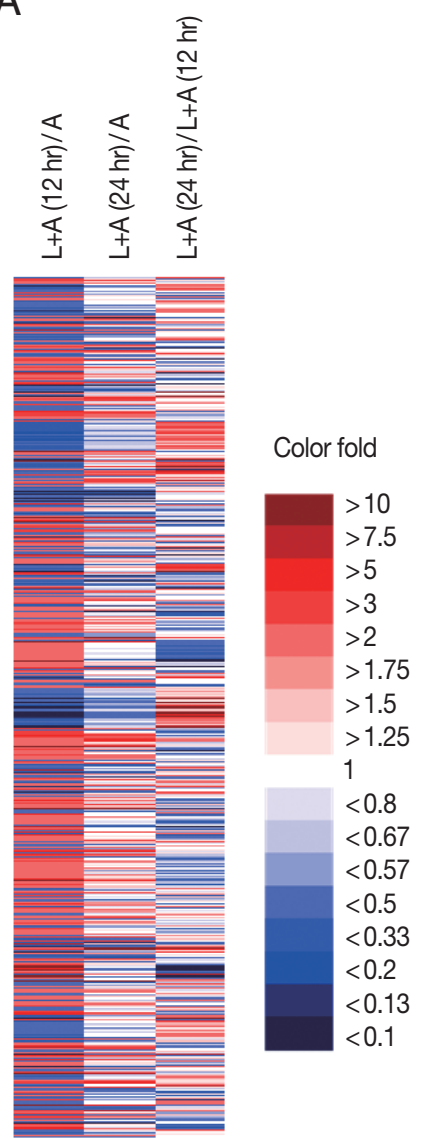

B

\begin{tabular}{cccc}
\hline & $\mathrm{L}+\mathrm{A}(12 \mathrm{hr}) / \mathrm{A}$ & $\mathrm{L}+\mathrm{A}(24 \mathrm{hr}) / \mathrm{A}$ & $\mathrm{L}+\mathrm{A}(24 \mathrm{hr}) / \mathrm{L}+\mathrm{A}(12 \mathrm{hr})$ \\
\hline $\begin{array}{c}\text { No. of } \\
\text { up-regulated } \\
\begin{array}{c}\text { No. of } \\
\text { down-regulated }\end{array}\end{array}$ & 1,211 & 1,321 & 996 \\
\hline
\end{tabular}

C

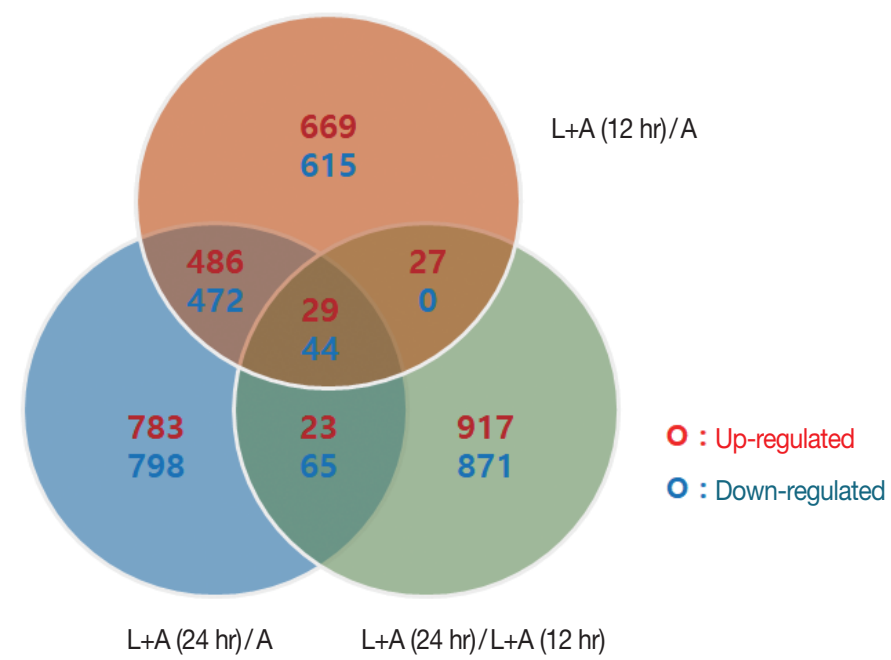

Fig. 1. An overview of significant changes in the gene expression profiles of $A$. castellanii. (A) Gene expressions under different experimental conditions displayed using a heat map. (B) The number of genes with significantly increased or decreased expression (more than 2 fold). (C) Venn diagram showing the number of overlapping genes differentially expressed among 3 experimental conditions. $L+A(12$ $\mathrm{hr}) / \mathrm{A}$; L. pneumohpila infected A. castellanii for $12 \mathrm{hr} / A$. castellanii, L+A(24 hr)/A; L. pneumohpila infected A. castellanii for $24 \mathrm{hr} / A$. castellanii, L+A(24 hr)/L+A(12 hr); L. pneumohpila infected $A$. castellanii for $24 \mathrm{hr} / \mathrm{L}$. pneumohpila infected $A$. castellanii for $12 \mathrm{hr}$.

ic activity (GO: 0003824). Based on these findings, it can be speculated that these DEGs may be of importance during the initial phase of infection. While xylosyltransferase 1 was downregulated more than 700 fold, sulfiredoxin 1 was downregulated more than 500 fold, and vacuolar sorting-associated protein 13 were downregulated more than 12 fold. (Table 2). GO analysis results revealed that the DEGs downregulated 10 fold or more were predominantly associated with the integral component of membrane (GO: 0016021). Findings are consistent with the changes in DEGs categorized under CC as illustrated in Fig. 3.

Although 132 out of 1,321 DEGs were upregulated and 54 out of 1,379 DEGs were downregulated more than 10 fold in A. castellanii $24 \mathrm{hr}$ pi, approximately $60 \%$ of these DEGs (78 DEGs and 30 DEGs) were identified as hypothetical proteins.
Strong inhibition of DEGs were observed in both $12 \mathrm{hr}$ pi A. castellanii (90 DEGs) and $24 \mathrm{hr}$ pi A. castellanii (53 DEGs). Among the DEGs demonstrating 10 fold or greater changes, 47 genes were upregulated while 90 genes were downregulated within the initial $12 \mathrm{hr}$ pi (Tables 1, 2). Conversely, by $24 \mathrm{hr}$ pi, 132 upregulated and 54 downregulated DEGs were observed. From these results, we supposed that $L$. pneumophila infection facilitated reduced A. castellanii gene expression during the early stage of infection to inhibit phagocytic digestion, while enhancing the expression of specialized A. castellanii genes during the late infection stage for LCV lysis and access to host cell machinery for intracellular replication.

Interestingly, L. pneumophila-infected A. castellanii showed differential expressions of methyltransferase-associated proteins. In addition to the S-adenosylmethionine-dependent 


\section{Biological process}

Mitochondrial respiratory chain... Metabolic process Aromatic amino acid family metabolic...

De novo' IMP biosynthetic process

Regulation of transcription, DNA-... Protein kinase C-activating G-protein...

DNA replication initiation Cell cyde

Response to stress DNA replication

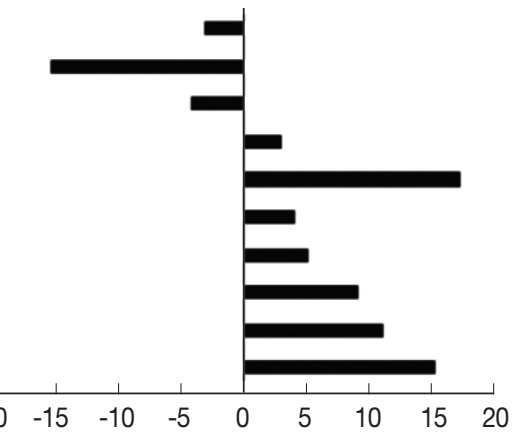

Cellular component

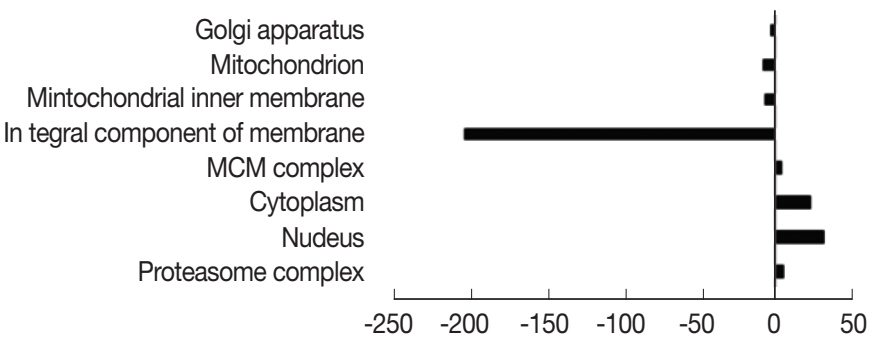

Molecular function

L-ascorbic acid binding Cytochrome-c oxidase activity Oxidoreductase activity Flavin adenine dinucleotide binding Oxidoreductase activity, acting on the...

Electron carrier activity Monooxygenase activity Heme binding 2 iron, 2 sulfur cluster binding Oxidoreductase activity, acting on paired... Iron ion binding

Microtubule motor activity Kinase activity FMN binding Hydrolase activity Diacylglycerol kinase activity NAD+binding Catalytic activity DNA-directed DNA polymerase activity Ligase activity DNA binding

NAD+ADP-ribosyltransferase activity Iron-sulfur duster binding

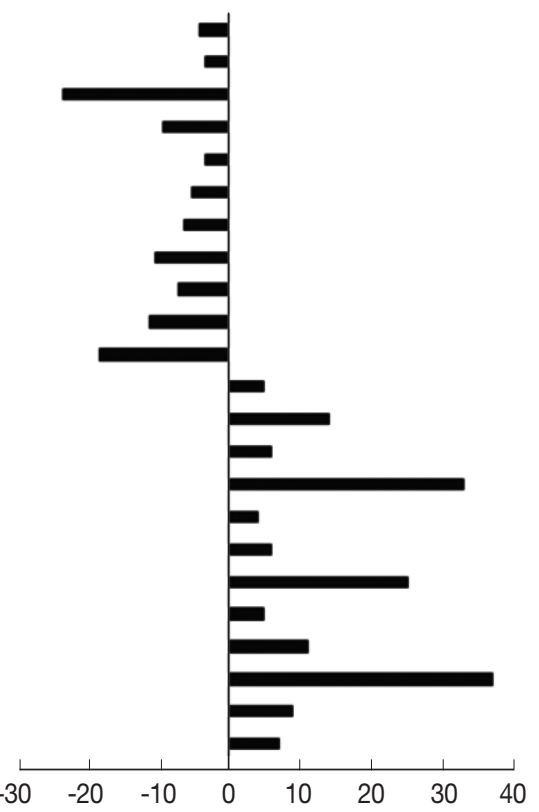

Fig. 2. Distribution of gene ontology (GO) functional classifications. GO analysis of downregulated (left-hand direction) and upregulated (right-hand direction) genes in A. castellanii infected with L. pneumophila after $12 \mathrm{hr}$.

methyltransferases and lysine methyltransferase enzyme domain-containing protein (Table 1), 11 DEGs associated with methyltransferase were upregulated, and 19 DEGs were down- regulated upon infection with $L$. pneumophila for $12 \mathrm{hr}$. Furthermore, L. pneumophila-infected A. castellanii also demonstrated differential expressions of acetyltransferase-associated 


\section{Biological process}

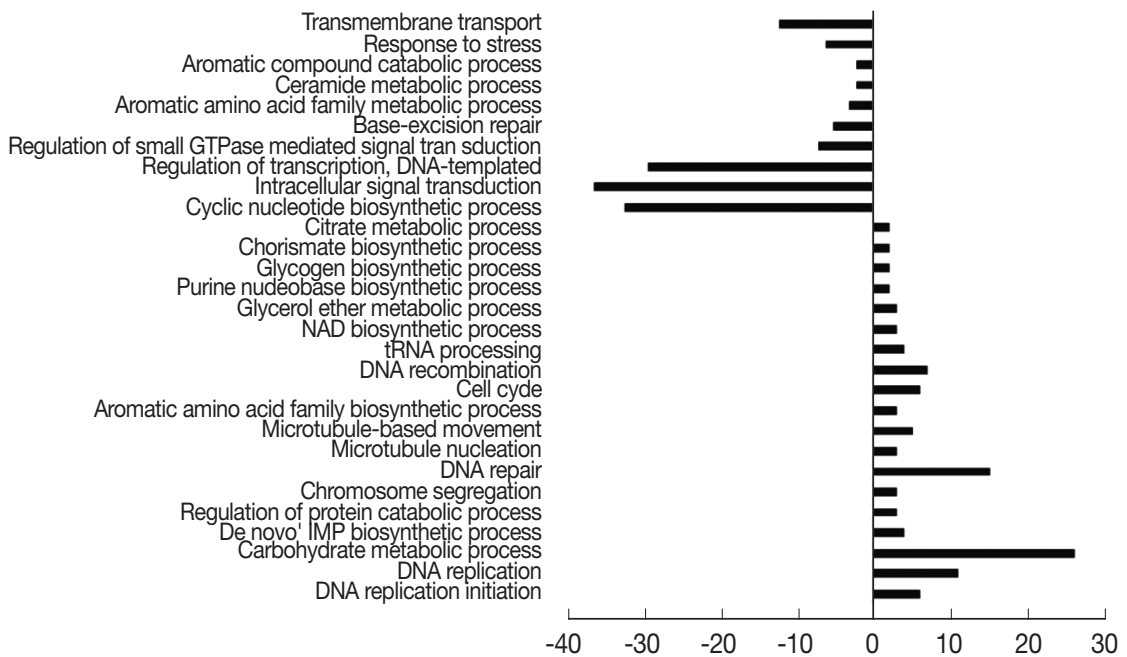

Cellular component

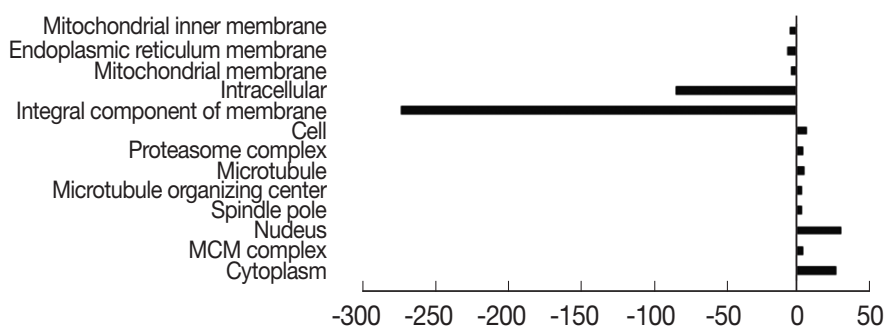

Molecular function

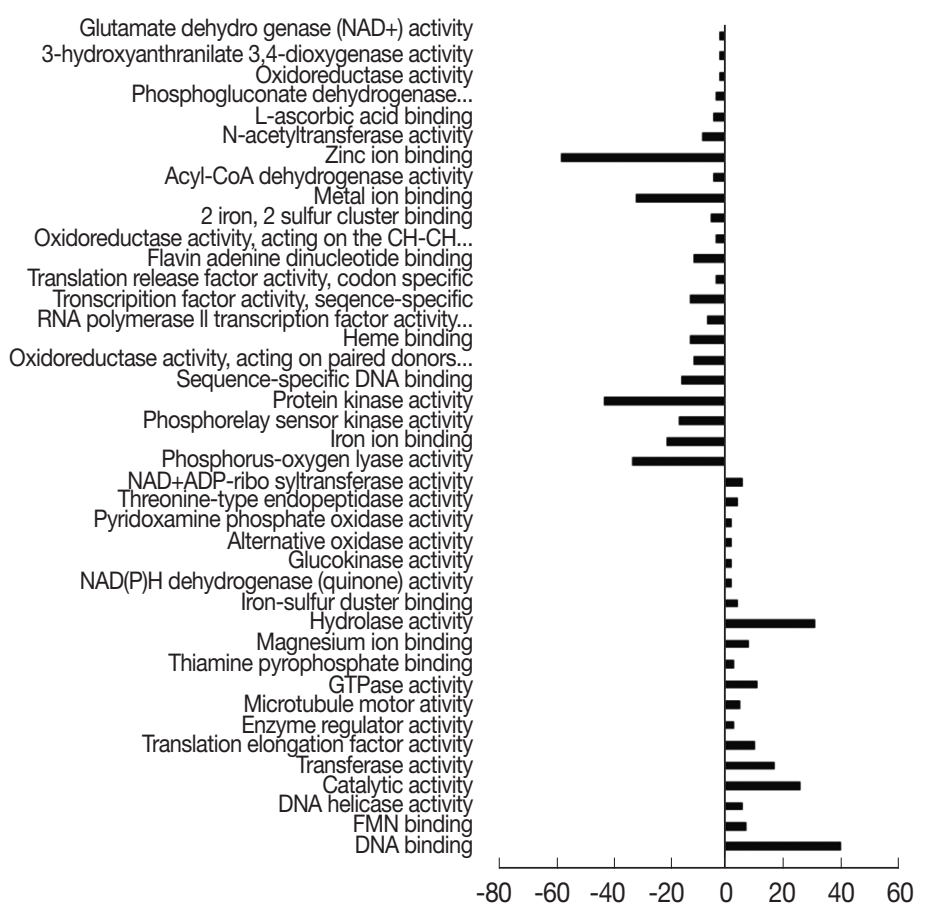

Fig. 3. Distribution of gene ontology (GO) functional classifications. GO analysis of downregulated (left-hand direction) and upregulated (right-hand direction) genes in A. castellanii infected with L. pneumophila after $24 \mathrm{hr}$. 
Table 1. Genes upregulated more than 10 fold in A. castellanii $12 \mathrm{hr}$ pi

\begin{tabular}{|c|c|c|c|c|}
\hline \multirow{2}{*}{ Gene symbol } & \multicolumn{2}{|c|}{ Fold change } & \multirow{2}{*}{$\begin{array}{c}\text { Annotation } \\
\text { Product }\end{array}$} & \multirow{2}{*}{$\begin{array}{c}\text { GO analysis } \\
\text { Category (Term) }\end{array}$} \\
\hline & $L+A(12) / A$ & $L+A(24) / A$ & & \\
\hline ACA1_328910 & 1099.734 & 136.04 & hypothetical protein & MF (GO:0003677) \\
\hline ACA1_183610 & 345.332 & 0.920 & hypothetical protein & - \\
\hline ACA1_183700 & 173.774 & 0.462 & S-adenosylmethionine-dependent methyltransferases & - \\
\hline ACA1_140050 & 116.896 & 15.154 & hypothetical protein & CC (GO:0016021) \\
\hline ACA1_183570 & 87.745 & 0.232 & GDPD-mannose-3',5'-epimerase & MF (GO:0003824) \\
\hline ACA1_324870 & 66.918 & 38.929 & hypothetical protein & - \\
\hline ACA1_300830 & 61.232 & 23.274 & permeases of the major facilitator superfamily & CC (GO:0016021) \\
\hline ACA1_139940 & 51.100 & 7.577 & hypothetical protein & - \\
\hline ACA1_183970 & 50.688 & 0.239 & hypothetical protein & CC (GO:0016021) \\
\hline ACA1_264780 & 41.374 & 2.313 & hypothetical protein & - \\
\hline ACA1_159010 & 36.741 & 11.383 & NmrAlike family protein & - \\
\hline ACA1_098380 & 27.710 & 0.838 & hypothetical protein & - \\
\hline ACA1_248200 & 25.965 & 3.365 & phosphotransferase enzyme domain containing protein & - \\
\hline ACA1_183940 & 25.733 & 0.905 & GTPase activating Rap/RanGAP domainlike 3, putative & - \\
\hline ACA1_096640 & 20.427 & 7.341 & hypothetical protein & - \\
\hline ACA1_183760 & 17.942 & 0.999 & lysine methyltransferase enzyme domain containing protein & - \\
\hline ACA1_376940 & 17.875 & 3.938 & BNR/Aspbox repeat domain containing protein & - \\
\hline ACA1_183580 & 17.602 & 0.724 & S-adenosylmethionine-dependent methyltransferases & - \\
\hline ACA1_270170 & 15.076 & 1.686 & von Willebrand factor type A domain containing protein & - \\
\hline ACA1_158840 & 15.058 & 6.332 & metal dependent phosphohydrolase & - \\
\hline ACA1_289630 & 14.765 & 11.367 & hypothetical protein & - \\
\hline ACA1_381540 & 14.601 & 0.503 & hypothetical protein & - \\
\hline ACA1_224160 & 14.371 & 6.761 & Sec23/Sec24 beta-sandwich domain containing protein & - \\
\hline ACA1_175370 & 14.029 & 6.388 & Erf4 domain containing protein & CC (GO:0016021) \\
\hline ACA1_140540 & 13.733 & 6.286 & MORN repeatcontaining protein & - \\
\hline ACA1_184710 & 13.687 & 26.561 & Phospholipid methyltransferase domain containing protein & CC (GO:0016021) \\
\hline ACA1_068540 & 13.266 & 5.034 & Prokumamolisin, activation domain containing protein & - \\
\hline ACA1_217750 & 13.172 & 4.030 & phosphoenolpyruvate carboxykinase (GTP), putative & MF (GO:0016301) \\
\hline ACA1_116700 & 13.149 & 9.620 & hypothetical protein & - \\
\hline ACA1_279740 & 12.960 & 5.501 & hydrogenase assembly factor, putative & MF (GO:0051536) \\
\hline ACA1_116690 & 12.553 & 5.917 & hypothetical protein & CC (GO:0016021) \\
\hline ACA1_285180 & 12.470 & 58.313 & DNA breaking-rejoining enzyme domain containing protein & MF (GO:0003677) \\
\hline ACA1_215790 & 12.170 & 8.113 & copper/zinc superoxide dismutase & - \\
\hline ACA1_275740 & 12.097 & 4.360 & glycerol-3-phosphate dehydrogenase (soluble) & MF (GO:0051287) \\
\hline ACA1_058320 & 12.094 & 4.914 & GPR1/FUN34/yaaH family protein & CC (GO:0016021) \\
\hline ACA1_358270 & 11.433 & 7.344 & pyridine nucleotidedisulfide oxidoreductase domain containing protein & MF (GO:0016491) \\
\hline ACA1_067720 & 11.300 & 8.046 & hypothetical protein & - \\
\hline ACA1_153710 & 11.001 & 0.845 & RFX_DNA_binding & $\mathrm{BP}(\mathrm{GO}: 0006355)$ \\
\hline ACA1_091110 & 10.950 & 0.842 & hypothetical protein & - \\
\hline ACA1_256560 & 10.936 & 3.671 & hypothetical protein & - \\
\hline ACA1_275730 & 10.900 & 2.743 & phosphoglycerate mutase family domain containing protein & - \\
\hline ACA1_165640 & 10.762 & 1.971 & hypothetical protein & - \\
\hline ACA1_060580 & 10.631 & 4.515 & phosphatase & - \\
\hline ACA1_245710 & 10.555 & 7.545 & hypothetical protein & - \\
\hline ACA1_325450 & 10.443 & 4.372 & CBS domain containing protein & - \\
\hline ACA1_187310 & 10.274 & 6.715 & heme NO binding domain containing protein & - \\
\hline ACA1_238590 & 10.109 & 3.454 & CBS domain containing protein & - \\
\hline
\end{tabular}

proteins. Histone acetyltransferase-associated protein was upregulated and 8 other acetyltransferases were downregulated $12 \mathrm{hr}$ pi with L. pneumophila. Icm/Dot type IV secretion system and its effectors of $L$. pneumophila modulate host gene expression by altering the chromatin structure or by affecting the activities of transcription factors [19]. Post-translational modifi- 
Table 2. Genes downregulated more than 10 fold in A. castellanii $12 \mathrm{hr}$ pi

\begin{tabular}{|c|c|c|c|c|}
\hline \multirow{2}{*}{ Gene symbol } & \multicolumn{2}{|c|}{ Fold change } & \multirow{2}{*}{$\begin{array}{c}\text { Annotation } \\
\text { Product }\end{array}$} & \multirow{2}{*}{$\begin{array}{c}\text { GO analysis } \\
\text { Category (Term) }\end{array}$} \\
\hline & $L+A(12) / A$ & $L+A(24) / A$ & & \\
\hline ACA1_113420 & 0.001 & 0.764 & hypothetical protein & - \\
\hline ACA1_112520 & 0.001 & 0.493 & Cysteine-rich 4 helical bundle widely conserved & - \\
\hline ACA1_111980 & 0.003 & 0.368 & EF hand domain containing protein & - \\
\hline ACA1_112090 & 0.003 & 0.454 & hypothetical protein & - \\
\hline ACA1_111740 & 0.007 & 0.190 & hypothetical protein & - \\
\hline ACA1_112480 & 0.007 & 0.492 & Fbox domain containing protein & - \\
\hline ACA1_376130 & 0.007 & 0.386 & xylosyltransferase 1, putative & - \\
\hline ACA1_058410 & 0.009 & 0.019 & hypothetical protein & - \\
\hline ACA1_147740 & 0.010 & 0.006 & CBS domain containing protein & - \\
\hline ACA1_113310 & 0.013 & 0.765 & hypothetical protein & - \\
\hline ACA1_166550 & 0.017 & 0.318 & hypothetical protein & - \\
\hline ACA1_374390 & 0.018 & 0.924 & hypothetical protein & - \\
\hline ACA1_101570 & 0.019 & 1.673 & hypothetical protein & - \\
\hline ACA1_392590 & 0.020 & 0.401 & hypothetical protein & - \\
\hline ACA1_060120 & 0.021 & 0.548 & hypothetical protein & - \\
\hline ACA1_400130 & 0.021 & 0.021 & hypothetical protein & - \\
\hline ACA1_307550 & 0.022 & 0.084 & Fbox domain containing protein & - \\
\hline ACA1_230230 & 0.022 & 0.022 & hypothetical protein & - \\
\hline ACA1_050390 & 0.024 & 2.233 & 3-oxoacyl-[acyl-carrier protein] reductase & CC (GO: 0016021) \\
\hline ACA1_112110 & 0.024 & 0.463 & glycosyl transferase & CC (GO: 0016021) \\
\hline ACA1_112490 & 0.024 & 0.480 & hypothetical protein & - \\
\hline ACA1_063680 & 0.026 & 0.072 & Reverse transcriptase & - \\
\hline ACA1_390590 & 0.027 & 0.132 & Hsp20/alpha crystallin superfamily protein & - \\
\hline ACA1_063960 & 0.029 & 0.078 & hypothetical protein & - \\
\hline ACA1_112130 & 0.029 & 0.457 & regulator of g protein signaling domain containing protein & - \\
\hline ACA1_111970 & 0.029 & 0.399 & sulfiredoxin 1 & - \\
\hline ACA1_158820 & 0.029 & 0.646 & hypothetical protein & - \\
\hline ACA1_064370 & 0.029 & 0.029 & AT Hook plus PHD finger transcription factor family member (athp1), putative & - \\
\hline ACA1_064780 & 0.029 & 0.029 & hypothetical protein & - \\
\hline ACA1_064790 & 0.029 & 0.029 & hypothetical protein & - \\
\hline ACA1_340040 & 0.030 & 0.275 & zinc finger, zz type domain containing protein & - \\
\hline ACA1_350050 & 0.030 & 0.298 & hypothetical protein & - \\
\hline ACA1_112530 & 0.031 & 0.499 & NLPC_P60 super family & - \\
\hline ACA1_112180 & 0.032 & 0.527 & major facilitator subfamily transporter & CC (GO: 0016021) \\
\hline ACA1_112590 & 0.034 & 0.474 & WH2 motif domain containing protein & - \\
\hline ACA1_050380 & 0.036 & 1.885 & hypothetical protein & - \\
\hline ACA1_230220 & 0.037 & 0.025 & hypothetical protein & - \\
\hline ACA1_199000 & 0.038 & 0.229 & SnoaL-like domain containing protein & - \\
\hline ACA1_077210 & 0.040 & 0.376 & hypothetical protein & - \\
\hline ACA1_173000 & 0.043 & 0.352 & Predicted NAD/FAD-dependent oxidoreductase & - \\
\hline ACA1_326260 & 0.043 & 0.073 & hypothetical protein & - \\
\hline ACA1_060740 & 0.045 & 0.310 & hypothetical protein & - \\
\hline ACA1_270160 & 0.046 & 0.071 & fascin subfamily protein & - \\
\hline ACA1_200180 & 0.048 & 0.428 & hypothetical protein & - \\
\hline ACA1_383480 & 0.051 & 0.029 & hypothetical protein & - \\
\hline ACA1_155760 & 0.052 & 0.145 & phosphoribosyltransferase & - \\
\hline ACA1_207830 & 0.052 & 0.426 & myotubularins and other putative membrane-associated proteins & - \\
\hline ACA1_133180 & 0.052 & 0.254 & Ser/Thr phosphatase family superfamily protein & - \\
\hline ACA1_077290 & 0.053 & 0.638 & 5'nucleotidase & CC (GO: 0016021) \\
\hline ACA1_365080 & 0.053 & 0.367 & hypothetical protein & - \\
\hline
\end{tabular}


Table 2. Continued

\begin{tabular}{|c|c|c|c|c|}
\hline \multirow{2}{*}{ Gene symbol } & \multicolumn{2}{|c|}{ Fold change } & \multirow{2}{*}{$\begin{array}{c}\text { Annotation } \\
\text { Product }\end{array}$} & \multirow{2}{*}{$\begin{array}{c}\text { GO analysis } \\
\text { Category (Term) }\end{array}$} \\
\hline & $L+A(12) / A$ & $L+A(24) / A$ & & \\
\hline ACA1_383650 & 0.055 & 0.349 & Human glyoxalase domain-containing protein 5 and similar proteins & - \\
\hline ACA1_048480 & 0.056 & 0.616 & hypothetical protein & - \\
\hline ACA1_055330 & 0.059 & 0.292 & N-terminal region of Chorein or VPS13 & - \\
\hline ACA1_346470 & 0.060 & 0.450 & RUN domain containing protein & - \\
\hline ACA1_298420 & 0.062 & 0.056 & hypothetical protein & - \\
\hline ACA1_197730 & 0.064 & 0.823 & hypothetical protein & - \\
\hline ACA1_378930 & 0.064 & 0.329 & hypothetical protein & - \\
\hline ACA1_214630 & 0.067 & 0.147 & hypothetical protein & CC (GO: 0016021) \\
\hline ACA1_128200 & 0.068 & 0.051 & CBS domain containing protein & - \\
\hline ACA1_052800 & 0.071 & 0.571 & O-methyltransferase family 3 protein & - \\
\hline ACA1_112560 & 0.071 & 0.499 & hypothetical protein & - \\
\hline ACA1_322750 & 0.072 & 0.552 & Glycosyl hydrolases family 2, TIM barrel domain & - \\
\hline ACA1_383400 & 0.072 & 1.376 & hypothetical protein & CC (GO: 0016021) \\
\hline ACA1_253630 & 0.073 & 0.947 & hypothetical protein & - \\
\hline ACA1_391470 & 0.074 & 0.821 & Hsp20/alpha crystallin superfamily protein & - \\
\hline ACA1_066110 & 0.074 & 0.05 & SCP-like extracellular protein domain containing protein & - \\
\hline ACA1_131790 & 0.075 & 0.133 & protein from patent family protein & - \\
\hline ACA1_111930 & 0.075 & 0.311 & carbonsulfur lyase, putative & BP (GO: 0008152) \\
\hline ACA1_064380 & 0.077 & 0.052 & hypothetical protein & - \\
\hline ACA1_064940 & 0.077 & 0.029 & Fbox domain containing protein & - \\
\hline ACA1_323370 & 0.077 & 0.011 & Hsp20/alpha crystallin superfamily protein & - \\
\hline ACA1_383750 & 0.078 & 0.236 & Ubiquitinconjugating enzyme subfamily protein & - \\
\hline ACA1_006080 & 0.079 & 0.210 & hypothetical protein & CC (GO: 0016021) \\
\hline ACA1_180590 & 0.082 & 0.546 & TRRAP family protein & - \\
\hline ACA1_112980 & 0.083 & 0.755 & protein kinase & - \\
\hline ACA1_077300 & 0.083 & 0.247 & serine/threonine kinase & CC (GO: 0016021) \\
\hline ACA1_111880 & 0.084 & 0.225 & Small acidic protein family & - \\
\hline ACA1_372720 & 0.084 & 0.148 & obtusifoliol 14alphademethylase, putative & CC (GO: 0016021) \\
\hline ACA1_046720 & 0.086 & 0.076 & Glycosyl hydrolase families & - \\
\hline ACA1_324050 & 0.088 & 0.646 & Vacuolar sorting-associated protein 13 [Intracellular trafficking and secretion] & - \\
\hline ACA1_400540 & 0.088 & 0.076 & sphingosine hydroxylase & CC (GO: 0016021) \\
\hline ACA1_290200 & 0.089 & 0.329 & hypothetical protein & - \\
\hline ACA1_389110 & 0.09 & 1.016 & hypothetical protein & - \\
\hline ACA1_112500 & 0.094 & 0.535 & TBC domain containing protein & - \\
\hline ACA1_311650 & 0.095 & 0.096 & hypothetical protein & - \\
\hline ACA1_112060 & 0.096 & 0.429 & O-methyltransferase, putative & - \\
\hline ACA1_178260 & 0.097 & 0.118 & cytochrome P450, putative & MF (GO: 0005506) \\
\hline ACA1_066960 & 0.098 & 1.036 & hypothetical protein & - \\
\hline ACA1_046710 & 0.099 & 0.126 & cytoplasmic protein, putative & - \\
\hline ACA1_112640 & 0.100 & 0.498 & MBOAT family protein & CC (GO: 0016021) \\
\hline
\end{tabular}

cations such as DNA methylation, histone acetylation, and histone methylation have been shown to play a critical role in the epigenetic regulation of eukaryotic gene expression $[19,20]$. Our results revealed that L. pneumophila-infected A. castellanii showed differential expressions of 30 kinds of methyltransferase-associated proteins and 9 kinds of acetyltransferase-associated proteins at $12 \mathrm{hr}$ pi. Based on the changes to epigenetic regulatory gene expressions, it can be speculated that L. pneumophila can alter the gene expression of A. castellanii through epigenetic mechanisms.

A plethora of DEGs induced in A. castellanii by the endosymbiont $L$. pneumophila were revealed in this study. However, $38.3 \%(1,930$ of the 5,042$)$ of A. castellanii genes were identified to be hypothetical proteins. Proportions of theses hypothetical proteins in the 12 and 24 hr pi groups can be ascribed to the lack of Acanthamoeba spp. database. Our investigation of 
the DEGs in A. castellanii by an endosymbiont provides important information to understanding the survival strategy utilized by notable intracellular pathogen $L$. pneumophila in $A$. castellanii. Future studies investigating the presence of an endosymbiosis-specific gene may help elucidate the underlying mechanism involved in L. pneumophila pathogenesis, which would contribute to understanding the inhibition of phagocytosis within A. castellanii or even immune evasion mechanism in human macrophages.

\section{ACKNOWLEDGMENT}

This work was supported by the National Research Foundation of Korea (NRF) grant funded by Korea government (MIST) (No. 2020R1F1A1068719).

\section{CONFLICT OF INTEREST}

The authors declare that they have no conflict of interest.

\section{REFERENCES}

1. Visvesvara GS, Moura H, Schuster FL. Pathogenic and opportunistic free-living amoebae: Acanthamoeba spp., Balamuthia mandrillaris, Naegleria fowleri, and Sappinia diploidea. FEMS Immunol Med Microbiol 2007; 50: 1-26. https://doi.org/10.1111/j.1574695X.2007.00232.X

2. Schmitz-Esser S, Toenshoff ER, Haider S, Heinz E, Hoenninger VM, Wagner M, Horn M. Diversity of bacterial endosymbionts of environmental Acanthamoeba isolates. Appl Environ Microbiol 2008; 74: 5822-5831. https://doi.org/10.1128/AEM.01093-08

3. Greub G, Raoult D. Microorganisms resistant to free-living amoebae. Clin Microbiol Rev 2004; 17: 413-433. https://doi.org/10.1128/ cmr.17.2.413-433.2004

4. Richards AM, Von Dwingelo JE, Price CT, Abu Kwaik Y. Cellular microbiology and molecular ecology of Legionella-amoeba interaction. Virulence 2013; 4: 307-314. https://doi.org/10.4161/ viru. 24290

5. Barker J, Brown MR. Trojan horses of the microbial world: protozoa and the survival of bacterial pathogens in the environment. Microbiology 1994; 140: 1253-1259. https://doi.org/10.1099/00221287140-6-1253

6. Essig A, Heinemann M, Simnacher U, Marre R. Infection of Acanthamoeba castellanii by Chlamydia pneumoniae. Appl Environ Microbiol 1997; 63: 1396-1399. https://doi.org/10.1128/AEM.63.4.13961399.1997

7. Guimaraes AJ, Gomes KX, Cortines JR, Peralta JM, Peralta RH. Acanthamoeba spp. as a universal host for pathogenic microorganisms: One bridge from environment to host virulence. Microbiol Res
2016; 193: 30-38. https://doi.org/10.1016/j.micres.2016.08.001

8. Isberg RR, O'Connor TJ, Heidtman M. The Legionella pneumophila replication vacuole: making a cosy niche inside host cells. Nat Rev Microbiol 2009; 7: 13-24. https://doi.org/10.1038/nrmicro1967

9. Vogel JP, Andrews HL, Wong SK, Isberg RR. Conjugative transfer by the virulence system of Legionella pneumophila. Science 1998; 279: 873-876. https://doi.org/10.1126/science.279.5352.873

10. Steiner B, Weber S, Hilbi H. Formation of the Legionella-containing vacuole: phosphoinositide conversion, GTPase modulation and ER dynamics. Int J Med Microbiol 2018; 308: 49-57. https:// doi.org/10.1016/j.ijmm.2017.08.004

11. Cazalet C, Rusniok C, Brüggemann H, Zidane N, Magnier A, Ma L, Tichit M, Jarraud S, Bouchier C, Vandenesch F, Kunst F, Etienne J, Glaser P, Buchrieser C. Evidence in the Legionella pneumophila genome for exploitation of host cell functions and high genome plasticity. Nat Genet 2004; 36: 1165-1173. https://doi.org/10.1038/ ng1447

12. Cazalet C, Gomez-Valero L, Rusniok C, Lomma M, Dervins-Ravault D, Newton HJ, Sansom FM, Jarraud S, Zidane N, Ma L, Bouchier C, Etienne J, Hartland EL, Buchrieser C. Analysis of the Legionella longbeachae genome and transcriptome uncovers unique strategies to cause Legionnaires' disease. PLoS Genet 2010; 6: e1000851. https://doi.org/10.1371/journal.pgen.1000851

13. Molmeret M, Bitar DM, Han L, Kwaik YA. Disruption of the phagosomal membrane and egress of Legionella pneumophila into the cytoplasm during the last stages of intracellular infection of macrophages and Acanthamoeba polyphaga. Infect Immun 2004; 72: 4040-4051. https://doi.org/10.1128/IAI.72.7.4040-4051.2004

14. Mou Q, Leung PHM. Differential expression of virulence genes in Legionella pneumophila growing in Acanthamoeba and human monocytes. Virulence 2018; 9: 185-196. https://doi.org/10.1080 /21505594.2017.1373925

15. Trapnell C, Pachter L, Salzberg SL. TopHat: discovering splice junctions with RNA-Seq. Bioinformatics 2009; 25: 1105-1111. https://doi.org/10.1093/bioinformatics/btp120

16. Quinlan AR, Hall IM. BEDTools: a flexible suite of utilities for comparing genomic features. Bioinformatics 2010; 26: 841-842. https://doi.org/10.1093/bioinformatics/btq033

17. Varet H, Brillet-Guéguen L, Coppée JY, Dillies MA. SARTools: A DESeq2- and EdgeR-Based R Pipeline for Comprehensive Differential Analysis of RNA-Seq Data. PLoS One 2016; 11: e0157022. https://doi.org/10.1371/journal.pone.0157022

18. Gentleman RC, Carey VJ, Bates DM, Bolstad B, Dettling M, Dudoit S, Ellis B, Gautier L, Ge Y, Gentry J, Hornik K, Hothorn T, Huber W, Iacus S, Irizarry R, Leisch F, Li C, Maechler M, Rossini AJ, Sawitzki G, Smith C, Smyth G, Tierney L, Yang JY, Zhang J. Bioconductor: open software development for computational biology and bioinformatics. Genome Biol 2004; 5: R80. https:// doi.org/10.1186/gb-2004-5-10-r80

19. Qiu J, Luo ZQ. Legionella and Coxiella effectors: strength in diversity and activity. Nat Rev Microbiol 2017; 15: 591-605. https://doi. org/10.1038/nrmicro.2017.67

20. Yen CY, Huang HW, Shu CW, Hou MF, Yuan SS, Wang HR, 
Chang YT, Farooqi AA, Tang JY, Chang HW. DNA methylation, histone acetylation and methylation of epigenetic modifications as a therapeutic approach for cancers. Cancer Lett 2016; 373: 185-192. https://doi.org/10.1016/j.canlet.2016.01.036 